\title{
molecules
}

ISSN 1420-3049

www.mdpi.com/journal/molecules

Article

\section{A New Synthetic Route to Original Sulfonamide Derivatives in 2-Trichloromethylquinazoline Series: A Structure-Activity Relationship Study of Antiplasmodial Activity}

\section{Nicolas Primas ${ }^{1}$, Pierre Verhaeghe ${ }^{1}$, Anita Cohen ${ }^{1,2}$, Charline Kieffer ${ }^{1}$, Aurélien Dumètre ${ }^{2}$,} Sébastien Hutter ${ }^{2}$, Sylvain Rault ${ }^{3}$, Pascal Rathelot ${ }^{1}$, Nadine Azas ${ }^{2}$ and Patrice Vanelle ${ }^{1, *}$

1 Laboratoire de Pharmacochimie Radicalaire, Faculté de Pharmacie, Institut de Chimie Radicalaire UMR CNRS 7273, Aix-Marseille Univ, 27 Boulevard Jean Moulin, 13385 Marseille cedex 05, France; E-Mails: nicolas.primas@univ-amu.fr (N.P.); pierre.verhaeghe@univ-amu.fr (P.V.); anita.cohen@univ-amu.fr (A.C.); charline.kieffer@ap-hm.fr (C.K.); pascal.rathelot@univ-amu.fr (P.R.)

2 Infections Parasitaires, Transmission, Physiopathologie et Thérapeutique, UMR MD3, Faculté de Pharmacie, Aix-Marseille Univ, 27 Boulevard Jean Moulin, 13385 Marseille Cedex 05, France; E-Mails: aurelien.dumetre@univ-amu.fr (A.D.); sebastien.hutter@univ-amu.fr (S.H.); nadine.azas@univ-amu.fr (N.A.)

3 Centre d'Etudes et de Recherche sur le Médicament de Normandie, UPRES EA 4258, FR CNRS 3038 INC3M, UFR des Sciences Pharmaceutiques, Université de Caen Basse-Normandie, Boulevard Becquerel, 14032 Caen Cedex, France; E-Mail: sylvain.rault@unicaen.fr

* Author to whom correspondence should be addressed; E-Mail: patrice.vanelle@univ-amu.fr; Tel.: +33-491-835-580; Fax: +33-491-794-677.

Received: 14 May 2012; in revised form: 25 June 2012 / Accepted: 26 June 2012 /

Published: 5 July 2012

\begin{abstract}
We report herein a simple and efficient two-step synthetic approach to new 2-trichloromethylquinazolines possessing a variously substituted sulfonamide group at position 4 used to prepare new quinazolines with antiparasitic properties. Thus, an original series of 20 derivatives was synthesized, which proved to be less-toxic than previously synthesized hits on the human HepG2 cell line, but did not display significant antiplasmodial activity. A brief Structure-Activity Relationship (SAR) evaluation shows that a more restricted conformational freedom is probably necessary for providing antiplasmodial activity.
\end{abstract}


Keywords: quinazoline; trichloromethyl group; sulfonamide; microwaves; antiplasmodial activity

\section{Introduction}

The quinazoline ring is an important molecular scaffold whose derivatives display a wide variety of pharmacological properties. Indeed, they were used as potent tyrosine kinase and cellular phosphorylation inhibitors [1], and they also act as ligands of benzodiazepine and GABA receptors in the central nervous system (CNS) [2] or as DNA binders [3]. Some of them exhibit remarkable activity as anticancer [4-6], antiviral [6-8], antibacterial [9] and anti-TB agents [10,11]. Moreover, popular drugs containing the quinazoline unit are available on the market. For example, erlotinib and gefitinib are used as epidermal growth factor receptor inhibitors in the treatment of several types of tumors [12], especially lung cancer $[13,14]$. In the other hand, prazosin acts as an $\alpha$-adrenergic blocker and is used to treat high blood pressure [15]. In continuation of our research program centered on the design and synthesis of original molecules with pharmacological properties [16-19], our group recently described the preparation of new 4-substituted-2-trichloromethylquinazoline derivatives which exhibit original in vitro antiplasmodial properties [20-24]. The promising results displayed by these recent studies prompted us to synthesize new derivatives in this 2-trichloromethylquinazoline series in order to identify both more potent antiplasmodial derivatives and less toxic ones, in order to increase the selectivity index (SI). With this objective, we focused on the synthesis of $N$-(quinazolin-4yl)benzenesulfonamides, taking into account that the sulfonamide group is well-known for its pharmaceutical interest in the field of anti-infectious substances.

\section{Results and Discussion}

\subsection{Synthesis}

Earlier works described the synthesis of $N$-(quinazolin-4-yl)benzenesulfonamides by nucleophilic substitution of 4-methoxyquinazoline by the sulfanilamide anion [9], by the construction of the quinazoline core from $N$-arylimidines and $N$-tosylisocyanodichloride [25] or by reacting $p$-toluenesulfonylisothiocyanate with 2-arylquinazoline-4(3H)-thione [26].

More recent publications report the access to these sulfonamides from the readily available 4-chloroquinazoline derivatives. Aryl-sulfonamides $\mathbf{2}$ in the presence of bases such as sodium hydride or potassium $t$-butoxide underwent nucleophilic substitution of 4-chloroquinazoline $\mathbf{1}$ and afforded the desired compound $\mathbf{3}$ in limited yields [27,28] (Scheme 1).

It is worth mentioning that this reaction was never attempted starting from 4-chloro-2-trichloromethylquinazoline [29]. Although the yields obtained with this strategy remained low, we tried to perform this reaction using $p$-toluenesulfonamide. Unfortunately, in our hands, whatever the operating conditions applied only traces of product were detected with LC-MS (Scheme 2). 
Scheme 1. Synthesis of sulfonamide 3 starting from 4-chloroquinazoline $\mathbf{1 .}$<smiles>COc1cc2nc(Cl)nc(Cl)c2cc1OCC(C)(C)CC(C)(C)O</smiles>

Scheme 2. Synthesis of sulfonamide 6 starting from 4-chloro-2-trichloromethylquinazoline (4).
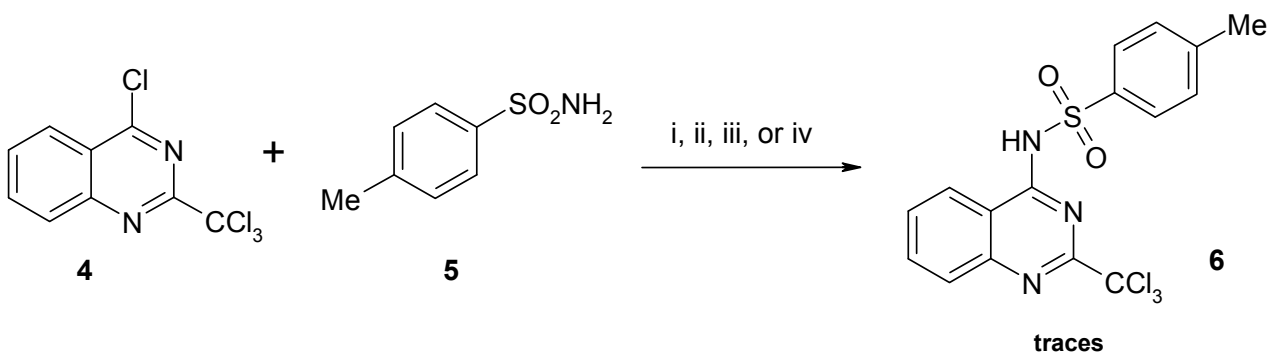

Reaction conditions: (i) $\mathrm{K}_{2} \mathrm{CO}_{3}$, PEG-400, $130{ }^{\circ} \mathrm{C}$, MW; (ii) $\mathrm{K}_{2} \mathrm{CO}_{3}$, DMF, $130{ }^{\circ} \mathrm{C}$, MW; (iii) $\mathrm{NaH}$, DMF, $80^{\circ} \mathrm{C}$; (iv) neat, $140{ }^{\circ} \mathrm{C}$.

Facing these failures, we chose to reverse the strategy by reacting readily available sulfonyl chlorides with 4-amino-2-trichloromethylquinazoline (7). We therefore investigated first of all the optimal synthesis of the key intermediate derivative 7 (Scheme 3).

Scheme 3. Synthesis of 4-amino-2-trichloromethylquinazoline (7).<smiles>Clc1nc(Cl)c2ccccc2n1</smiles>

4

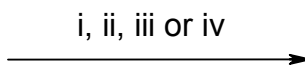

7

Reaction conditions: (i) $\mathrm{NH}_{4} \mathrm{OH} 25 \%$ in $\mathrm{H}_{2} \mathrm{O}$, traces; (ii) $\mathrm{NH}_{3} 2 \mathrm{M}$ in $\mathrm{MeOH}, 3$ days, rt, $91 \%$ (Method A); (iii) $\mathrm{NH}_{3} 0.5 \mathrm{M}$ in dioxane, $140{ }^{\circ} \mathrm{C}, 15 \mathrm{~min}, \mathrm{MW}, 44 \%$; (iv) $\mathrm{NH}_{3} 0.4 \mathrm{M}$ in THF, $140{ }^{\circ} \mathrm{C}$, 15 min, MW, 100\% (Method B).

The reaction of 4-chloro-2-trichloromethylquinazoline (4) with 25\% aqueous ammonium hydroxide led to the predominant formation of 2-trichloromethylquinazolin-4(3H)-one [23] accompanied by traces of the expected amino derivative 7, so it appeared that it was necessary to operate under anhydrous conditions to prevent hydrolysis. Thus, the use of a solution of $2 \mathrm{~N}$ ammonia in dry methanol afforded the expected amino derivative 7 in $71 \%$ yield after $24 \mathrm{~h}$ at room temperature. Increasing the time of the reaction up to $72 \mathrm{~h}$ increased the yield to $91 \%$ (Method A), while elevating the temperature with a view to shortening reaction time was detrimental and led mainly to the formation of 4-methoxy-2-trichloromethylquinazoline. In order to prevent this side reaction, due to the nucleophilic character of methanol, the amination must be conducted in a solvent inert towards the starting material 4. In order to shorten the reaction time we investigated the benefit of using sealed vials under microwave irradiation. Thus, under such conditions, the use of ammonia in dioxane ( $0.5 \mathrm{M}$, 
3 equiv.) afforded 7 in only $44 \%$ yield at $140{ }^{\circ} \mathrm{C}$. Finally, we found that heating 4 under the same conditions in THF ( $0.4 \mathrm{M}, 3$ equiv.) led to 7 in quantitative yield after only 15 min (Method B) which is not surprising given the known ability of THF to absorb microwaves.

Having secured good access to this key intermediate 4-amino-2-trichloromethylquinazoline (7), we next synthesized the target sulfonamides using variously substituted sulfonyl chlorides (Scheme 4) with a view to further study the influence of the substituent nature on the biological properties. Amino derivative 7 was reacted with an excess of sodium hydride in THF. The so-formed anion was then trapped with sulfonyl chloride. The resulting sulfonamides 8 were thus obtained in good yields (Table 1) without requiring any chromatographic purification (except for compound $\mathbf{8 t}$ ).

Scheme 4. Synthesis of $N$-(2-trichloromethylquinazolin-4-yl)sulfonamides 8.

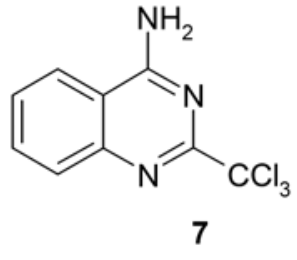

1) $\mathrm{NaH}, 5$ equiv. $0^{\circ} \mathrm{C}$ then $\mathrm{rt}$<smiles>[R]S(=O)(=O)Nc1nc(C(Cl)(Cl)Cl)nc2ccccc12</smiles>

$\mathrm{R}=\mathrm{Me}, \mathrm{Ar}$

Table 1. Reaction yields and biological evaluation for sulfonamides 8a-t.

\begin{tabular}{|c|c|c|c|c|c|}
\hline Entry & R- & Product & Yield \% & $\mathrm{CC}_{50}(\mu \mathrm{M})^{\mathrm{a}}$ & $\mathrm{IC}_{50} \mathrm{PfK} 1(\mu \mathrm{M})^{\mathrm{b}}$ \\
\hline 1 & $\mathrm{Ph}-$ & $8 a$ & 62 & 82.0 & $>10 *$ \\
\hline 2 & 4-Me-Ph- & $8 b$ & 51 & 68.7 & $>10 *$ \\
\hline 3 & 4-MeO-Ph- & $8 c$ & 56 & 102.3 & $>10 *$ \\
\hline 4 & 4-CN-Ph- & 8d & 73 & 73.8 & $>10 *$ \\
\hline 5 & 4-Cl-Ph & $8 \mathbf{e}$ & 50 & 71.1 & $>10 *$ \\
\hline 6 & 4-Br-Ph- & $8 f$ & 60 & 57.4 & $>10 *$ \\
\hline 7 & 4-F-Ph- & $8 g$ & 66 & 69.5 & $>10 *$ \\
\hline 8 & 3-F-Ph- & $8 \mathrm{~h}$ & 53 & 106.4 & $>10 *$ \\
\hline 9 & 2-F-Ph- & $8 \mathbf{i}$ & 60 & 136.1 & $>10 *$ \\
\hline 10 & $4-\mathrm{NO}_{2}-\mathrm{Ph}-$ & $8 \mathbf{j}$ & 91 & 88.3 & $>10 *$ \\
\hline 11 & $3-\mathrm{NO}_{2}-\mathrm{Ph}-$ & $8 \mathbf{k}$ & 56 & 119.8 & $>10 *$ \\
\hline 12 & $2-\mathrm{NO}_{2}-\mathrm{Ph}-$ & 81 & 59 & 126.0 & $>10 *$ \\
\hline 13 & 4- $\mathrm{CF}_{3}-\mathrm{Ph}-$ & $8 m$ & 88 & 49.0 & $>10 *$ \\
\hline 14 & $3-\mathrm{CF}_{3}-\mathrm{Ph}-$ & $8 n$ & 100 & 75.1 & $>10 *$ \\
\hline 15 & $2-\mathrm{CF}_{3}-\mathrm{Ph}-$ & 80 & 50 & 73.8 & $>10 *$ \\
\hline 16 & Biphenyl-4-yl & $8 p$ & 23 & 38.2 & $>10 *$ \\
\hline 17 & Napht-2-yl- & $8 q$ & 43 & 48.8 & $>10 *$ \\
\hline 18 & Napht-1-yl- & $8 r$ & 61 & 47.8 & $>10 *$ \\
\hline 19 & Thiophen-2-yl- & $8 s$ & 43 & 121.1 & $>10 *$ \\
\hline 20 & Methyl- & $8 t$ & 18 & 241.2 & $>10 *$ \\
\hline \multicolumn{4}{|c|}{ Doxorubicine $^{\mathrm{a}}$} & 0.2 & - \\
\hline \multicolumn{4}{|c|}{ Chloroquine $^{\mathrm{b}}$} & 30 & 0.6 \\
\hline \multicolumn{4}{|c|}{ Doxycycline $^{b}$} & 20 & 6.0 \\
\hline
\end{tabular}

${ }^{\mathrm{a}}$ Doxorubicine was used as a cytotoxic reference-drug; ${ }^{\mathrm{b}}$ chloroquinine and doxycycline were used as antimalarial reference-drugs; * Tested compounds did not show significant antiplasmodial activity at the highest tested concentration $(10 \mu \mathrm{M})$. 
Our efficient two-step methodology afforded the expected compounds in a $50-100 \%$ range yield except for the biphenyl-4-yl-, napht-2-yl, thiophen-2-yl and the methyl derivatives (compounds 8p, $\mathbf{8 q}, \mathbf{8 s}$ and 8t, respectively, entries 16, 17, 19 and 20) which were not obtained from simple benzenesulfonyl chlorides. One the one hand, the electron withdrawing or donating character of the substituent of benzenesulfonyl chlorides did not affect appear to notably the reaction yield. However, entries 4,10,13 and 14 corresponding to electron withdrawing groups are the ones which gave the best yields. On the other hand, the lowest reaction yields are attributable to the purification process (namely the high solubility of molecules $\mathbf{8 p}-\mathbf{t}$ in $\mathrm{CH}_{2} \mathrm{Cl}_{2}$ ).

\subsection{Biological Evaluation and Structure-Activity Relationship (SAR) Study}

Following previously reported experimental procedures [22,23], the in vitro antiplasmodial activity toward the multi-resistant W2 or K1 Plasmodium falciparum strains was measured by determining the $\mathrm{IC}_{50}$ (inhibition concentration $50 \%$ in $\mu \mathrm{M}$ ) and comparing them with two commercial reference-drugs: chloroquine and doxycycline. The cytotoxicity was measured toward the human HepG2 cell line by determining the $\mathrm{CC}_{50}$ (cytotoxic concentration $50 \%$ in $\mu \mathrm{M}$ ) and comparing them with doxorubicine used as a cytotoxic reference-drug. Concerning the biological evaluation, this series showed excellent solubility in biological media, contrary to the 4-thiophenoxy- series [22] whose cytotoxic evaluation was limited by a lack of solubility (Table 2 , entry 4 ).

Table 2. Antiplasmodial and cytotoxicity evaluation in 2-trichloromethylquinazoline series.

\begin{tabular}{|c|c|c|c|c|}
\hline Entry & Structure & $\mathrm{CC}_{50}$ HepG2 $(\mu \mathrm{M})$ & $\mathrm{IC}_{50} P$. falc. $(\mu \mathrm{M})$ & $\mathrm{SI}^{\mathrm{a}}$ \\
\hline 1 & & $>125$ & 2.5 & $>50$ \\
\hline 2 & & 16 & 0.4 & 40 \\
\hline 3 & & 50 & 1.1 & 45 \\
\hline
\end{tabular}


Table 2. Cont.

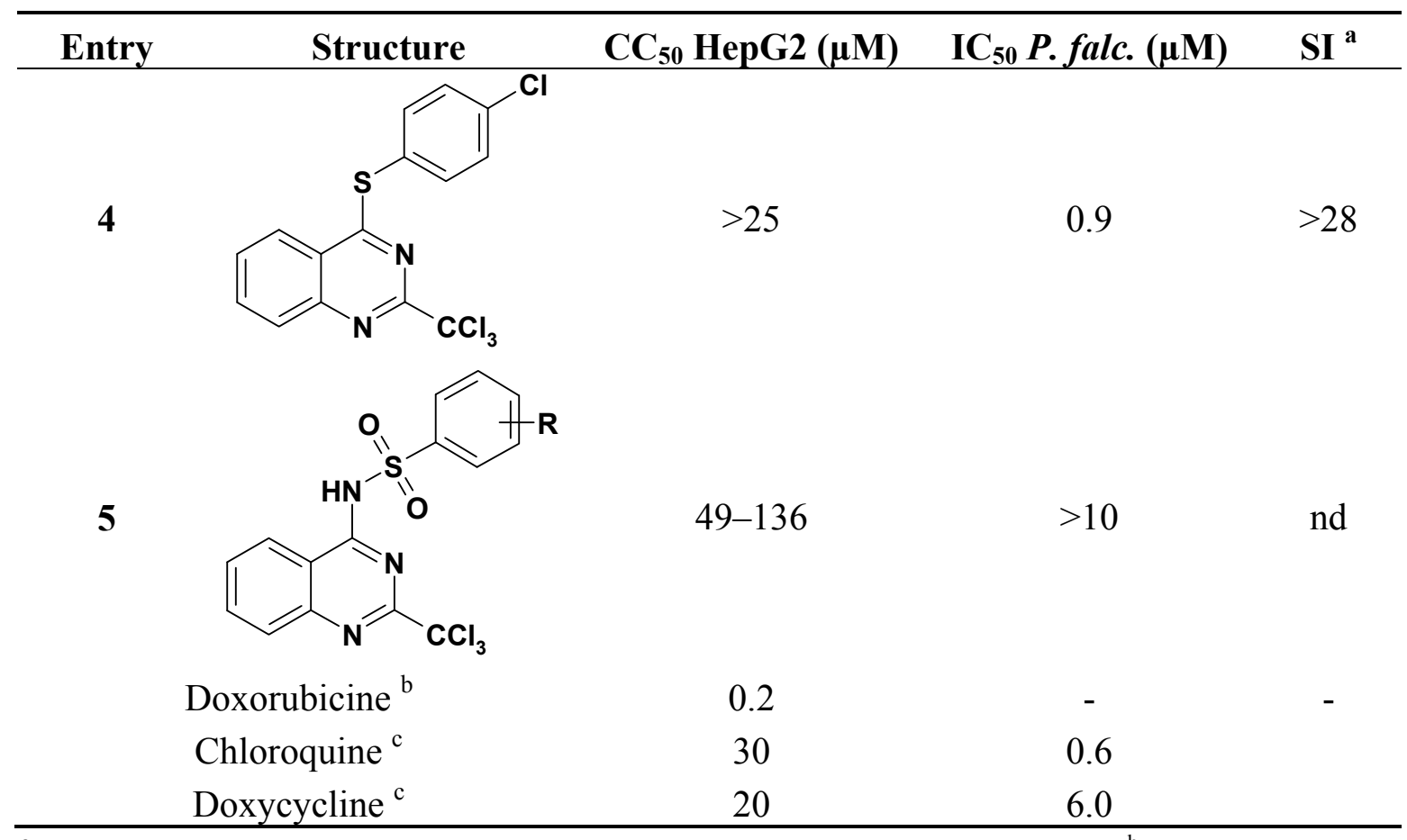

${ }^{\mathrm{a}}$ Selectivity index (SI) was calculated according to the formula: $\mathrm{SI}=\mathrm{CC}_{50} / \mathrm{IC}_{50}$; ${ }^{\mathrm{b}}$ Doxorubicine was used as a cytotoxic reference-drug; ${ }^{\mathrm{c}}$ Chloroquinine and Doxycycline were used as antimalarial reference-drugs.

Unfortunately, contrary to the previously synthesized series (Table 2, entries 1-4), the sulfonamide derivatives (Table 1 and Table 2, entry 5) did not display significant antiplasmodial activity with $\mathrm{IC}_{50}$ values $>10 \mu \mathrm{M}$. The Structure-Activity Relationship study seems to indicate that the introduction of a sulfonamide group at position 4 of the quinazoline ring leads to a larger gap between the phenyl substituent and the quinazoline ring. This structural change added a more important conformational freedom and reduced antiplasmodial activity. The most potent antiplasmodial molecule previously synthesized (Table 2, entry 2) displayed slight cytotoxicity $(16 \mu \mathrm{M})$ on the HepG2 human cell line which limits its selectivity index to 40. Concerning the sulfonamide derivatives, apart from the biphenyl derivative 8p (Table 1), this series appeared less-toxic than previously synthesized hits on the same cell line.

Thus, taking advantage of this brief SAR evaluation, two new 4-substituted quinazoline series presenting a more restricted conformational freedom (Figure 1) could be prepared by using the synthetic approach presented herein, in order to identify new antiplasmodial hits.

Figure 1. Novel investigated derivatives.<smiles>[R]c1cccc(/C=N/c2nc(C(Cl)(Cl)Cl)nc3ccccc23)c1</smiles> 


\section{Experimental}

\subsection{General Procedures}

Commercial reagents were used as received without additional purification. Melting points were determined on a Köfler melting point apparatus and are uncorrected. Elemental analyses and HRMS were carried out at the Spectropole, Faculté des Sciences et Techniques de Saint-Jérôme, Marseille, France. NMR spectra were recorded on a Bruker ARX 200 spectrometer at the Faculté de Pharmacie de Marseille $\left(200 \mathrm{MHz}{ }^{1} \mathrm{H}-\mathrm{NMR}\right.$ : reference $\mathrm{CHCl}_{3} \delta=7.26$, DMSO- $d_{6} \delta=2.50$ and $50 \mathrm{MHz}{ }^{13} \mathrm{C}$ : reference $\mathrm{CHCl}_{3} \delta=76.9$, DMSO- $\left.d_{6} \delta=39.5\right)$. Solvents were dried by conventional methods. The following adsorbent was used for column chromatography: silica gel 60 (Merck, particle size 0.063-0.200 mm, 70-230 mesh ASTM). TLC was performed on $5 \mathrm{~cm} \times 10 \mathrm{~cm}$ aluminium plates coated with silica gel 60F-254 (Merck) in an appropriate eluent. Visualization was made with ultraviolet light (234 nm). HRMS spectra were recorded on QStar Elite (Applied Biosystems SCIEX) spectrometer. PEG was the matrix for HRMS. The experimental exact mass was given for the ion which has the maximum isotopic abundance. Purity of synthetized compounds was checked with LC-MS analyses which were realized at the Faculty of Pharmacy of Marseille with a Thermo Scientific Accela High Speed LC System ${ }^{\circledR}$ coupled with a single quadrupole mass spectrometer Thermo MSQ Plus $^{\circledR}$. The RP-HPLC column used is a Thermo Hypersil Gold ${ }^{\circledR} 50 \times 2.1 \mathrm{~mm}$ (C18 bounded), with particles of $1.9 \mu \mathrm{m}$ diameter. The volume of sample injected on the column was $1 \mu \mathrm{L}$. The chromatographic analysis, total duration of $8 \mathrm{~min}$, is made with the gradient of following solvents: $\mathrm{t}=0 \mathrm{~min}$, water/methanol 50/50;0<t $<4 \mathrm{~min}$, linear increase in the proportion of water to a ratio water/methanol 95/5; $4<\mathrm{t}<6 \mathrm{~min}$, water/methanol 95/5; $6<\mathrm{t}<7 \mathrm{~min}$, linear decrease in the proportion of water to return to a ratio 50/50 water/methanol; $6<\mathrm{t}<7 \mathrm{~min}$, water/methanol 50/50. The water used was buffered with $5 \mathrm{mM}$ ammonium acetate. The retention times ( $\mathrm{Tr})$ of the molecules analyzed are indicated in min. The microwave reactions were performed using a Biotage Initiator Microwave oven using 10-20 mL sealed vials; temperatures were measured with an IR-sensor and reaction times given as hold times. The preparation of 4-chloro-2-trichloromethylquinazoline (4) was achieved as described in the literature [29].

\subsection{Preparation of 4-Amino-2-trichloromethylquinazoline (7)}

Method (A) To a stirred solution of 4-chloro-2-trichloromethylquinazoline (4, $2.0 \mathrm{~g}, 7.1 \mathrm{mmol})$ in dry THF $(10 \mathrm{~mL})$ under nitrogen at $\mathrm{rt}$, was added ammonia solution in methanol $(2.0 \mathrm{M}, 10.6 \mathrm{~mL}$, $21.3 \mathrm{mmol}, 3$ equiv.). The resulting mixture was stirred at this temperature for 3 days, and then, the volatiles were removed under vacuum. The residue was triturated with dichloromethane and filtered to give 7 as a white solid $(1.7 \mathrm{~g}, 91 \%)$.

Method (B) In a microwave vial equipped with a magnetic stir bar, 4-chloro-2trichloromethylquinazoline $(4,700 \mathrm{mg}, 2.48 \mathrm{mmol})$ and ammonia solution in THF $(0.4 \mathrm{M}, 18.6 \mathrm{~mL}$, $7.45 \mathrm{mmol}, 3$ equiv.). The vial was capped and the suspension was then heated at $140{ }^{\circ} \mathrm{C}$ for $15 \mathrm{~min}$ ( 8 bar). The volatiles were removed under vacuum. The residue was poured into EtOAc $(100 \mathrm{~mL})$ and extracted two times with brine $(30 \mathrm{~mL})$. The organic layer was dried with $\mathrm{Na}_{2} \mathrm{SO}_{4}$, filtered and evaporated to afford 2 as white solid (650 mg, 100\%). M.p. $199{ }^{\circ} \mathrm{C} .{ }^{1} \mathrm{H}-\mathrm{NMR}$ (DMSO- $\left.d_{6}\right)$ : $\delta$ 8.33-8.27 
(m, 3H), 7.90-7.77 (m, 2H), 7.65-7.57 (m, 1H). ${ }^{13} \mathrm{C}-\mathrm{NMR}$ (DMSO- $\left.d_{6}\right): \delta 163.2,160.9,148.8,134.1$, 128.3, 127.5, 123.9, 113.4, 98.4. LC-MS (ESI) Tr $2.37 \mathrm{~min}, m / z[\mathrm{M}+\mathrm{H}]^{+}$Calcd: 261.96, Found: 262.03. Anal. calcd. for $\mathrm{C}_{9} \mathrm{H}_{6} \mathrm{Cl}_{3} \mathrm{~N}_{3}$ : C, 41.18; H, 2.30; N, 16.01. Found: C, 41.43; H, 2.31; N, 16.29.

\subsection{General Procedure for the Preparation of Compounds $\mathbf{8 a}$ to $\mathbf{8 t}$}

To a slurry of $60 \%$ sodium hydride in oil $(152 \mathrm{mg}, 3.81 \mathrm{mmol}, 5$ equiv. $)$ in dry THF $(4 \mathrm{~mL})$ at $0{ }^{\circ} \mathrm{C}$ was slowly added 4-amino-2-trichloromethylquinazoline (7, $200 \mathrm{mg}, 0.76 \mathrm{mmol}, 1$ equiv.). After $30 \mathrm{~min}$ of stirring at $\mathrm{rt}$, the mixture was cooled again and the appropriate sulfonyl chloride $(1.52 \mathrm{mmol}$, 2 equiv.) was added portionwise. After this addition, the reaction was then stirred at $\mathrm{rt}$ until the disappearance of starting material. The excess of $\mathrm{NaH}$ was hydrolyzed with ice at $0{ }^{\circ} \mathrm{C}$. The mixture was extracted with EtOAc and washed three times with brine. The organic layer was dried with $\mathrm{Na}_{2} \mathrm{SO}_{4}$, filtered and evaporated. The crude residue was triturated in dichloromethane and filtered to give the corresponding sulfonamide $\mathbf{8}$.

$N$-(2-Trichloromethylquinazolin-4-yl)benzenesulfonamide (8a). White solid (62\%). M.p. $200{ }^{\circ} \mathrm{C}$. ${ }^{1} \mathrm{H}-\mathrm{NMR}$ (DMSO- $d_{6}$ ): $\delta 8.27(\mathrm{~d}, J=8.0 \mathrm{~Hz}, 1 \mathrm{H}), 8.02(\mathrm{t}, J=3.5 \mathrm{~Hz}, 2 \mathrm{H}), 7.75-7.60(\mathrm{~m}, 2 \mathrm{H}), 7.48$ (t, $J=7.0 \mathrm{~Hz}, 1 \mathrm{H}), 7.38-7.35$ (m, 3H). ${ }^{13} \mathrm{C}-\mathrm{NMR}$ (DMSO- $\left.d_{6}\right): \delta 163.1,160.3,148.6,144.8,132.5,130.0$, 127.8, 127.5, 127.1, 126.4, 125.4, 119.1, 98.9. LC-MS (ESI) Tr $1.36 \mathrm{~min}, \mathrm{~m} / z[\mathrm{M}+\mathrm{H}]^{+}$Calcd: 401.96, Found: 401.96. HRMS (ESI+) $m / z 423.9454[\mathrm{M}+\mathrm{Na}]^{+}$, calcd. for $\mathrm{C}_{15} \mathrm{H}_{10} \mathrm{~N}_{3} \mathrm{O}_{2} \mathrm{SCl}_{3}: 423.9452[\mathrm{M}+\mathrm{Na}]^{+}$.

4-Methyl-N-(2-trichloromethylquinazolin-4-yl)benzenesulfonamide (8b). White solid (51\%). M.p. $>260{ }^{\circ} \mathrm{C} .{ }^{1} \mathrm{H}-\mathrm{NMR}\left(\mathrm{DMSO}-d_{6}\right): \delta 8.24(\mathrm{~d}, J=7.8 \mathrm{~Hz}, 1 \mathrm{H}), 7.93(\mathrm{~d}, J=7.6 \mathrm{~Hz}, 2 \mathrm{H}), 7.69-7.58$ $(\mathrm{m}, 2 \mathrm{H}) ; 7.45(\mathrm{t}, J=7.6 \mathrm{~Hz}, 1 \mathrm{H}), 7.15(\mathrm{~d}, J=7.6 \mathrm{~Hz}, 2 \mathrm{H}), 2.27$ (s, 3H). ${ }^{13} \mathrm{C}-\mathrm{NMR}$ (DMSO- $\left.d_{6}\right): \delta$ 163.2, 160.5, 148.6, 142.2, 139.6, 132.3; 127.9, 127.8, 127.0, 126.2, 125.4, 119.4, 99.1; 20.9. LC-MS (ESI) Tr $1.98 \mathrm{~min}, m / z$ [M-H] ${ }^{-}$Calcd: 413.97, Found: 414.35. HRMS (ESI+) $m / z 437.9607$ [M+Na] calcd. for $\mathrm{C}_{16} \mathrm{H}_{12} \mathrm{~N}_{3} \mathrm{O}_{2} \mathrm{SCl}_{3}: 437.9608[\mathrm{M}+\mathrm{Na}]^{+}$.

4-Methoxy-N-(2-trichloromethylquinazolin-4-yl)benzenesulfonamide (8c). White solid (56\%). M.p. $196{ }^{\circ} \mathrm{C}$. ${ }^{1} \mathrm{H}-\mathrm{NMR}\left(\mathrm{DMSO}-d_{6}\right): \delta 8.23(\mathrm{~d}, J=7.8 \mathrm{~Hz}, 1 \mathrm{H}), 7.99(\mathrm{~d}, J=8.4 \mathrm{~Hz}, 2 \mathrm{H}), 7.69-7.61(\mathrm{~m}, 2 \mathrm{H}) ; 7.45(\mathrm{t}$, $J=7.2 \mathrm{~Hz}, 1 \mathrm{H}), 6.87(\mathrm{~d}, J=8.4 \mathrm{~Hz}, 2 \mathrm{H}), 3.73(\mathrm{~s}, 3 \mathrm{H}) .{ }^{13} \mathrm{C}-\mathrm{NMR}\left(\mathrm{DMSO}-d_{6}\right): \delta 163.1,160.5,148.6$, 137.2, 132.2, 129.8; 127.0, 126.2, 125.4, 119.4, 112.5, 99.1; 55.3. LC-MS (ESI) Tr $1.29 \mathrm{~min}, \mathrm{~m} / \mathrm{z}$ $[\mathrm{M}+\mathrm{H}]^{+}$Calcd: 431.97, Found: 431.90. HRMS (ESI+) $m / z 453.9559[\mathrm{M}+\mathrm{Na}]^{+}$, calcd. for $\mathrm{C}_{16} \mathrm{H}_{12} \mathrm{~N}_{3} \mathrm{O}_{3} \mathrm{SCl}_{3}: 453.9557[\mathrm{M}+\mathrm{Na}]^{+}$.

4-Cyano-N-(2-trichloromethylquinazolin-4-yl)benzenesulfonamide (8d). Beige solid (73\%). M.p. $204{ }^{\circ} \mathrm{C}$. ${ }^{1} \mathrm{H}-\mathrm{NMR}\left(\mathrm{DMSO}-d_{6}\right): \delta 8.34(\mathrm{~d}, J=7.8 \mathrm{~Hz}, 1 \mathrm{H}), 8.15(\mathrm{~d}, J=8.2 \mathrm{~Hz}, 2 \mathrm{H}), 7.87(\mathrm{~d}, J=8.4 \mathrm{~Hz}, 2 \mathrm{H})$, $7.82-7.67(\mathrm{~m}, 2 \mathrm{H}) ; 7.55$ (dt, $J=1.5 \mathrm{~Hz}, J=8.2 \mathrm{~Hz}, 1 \mathrm{H}) .{ }^{13} \mathrm{C}-\mathrm{NMR}$ (DMSO- $d_{6}$ ): $\delta 162.6,159.8,148.8$, 148.6, 133.2, 132.1, 128.4; 127.4, 127.1, 125.2, 118.4, 118.3, 112.8, 98.3. LC-MS (ESI) Tr 1.19 min, $m / z[\mathrm{M}-\mathrm{H}]^{-}$Calcd: 424.95, Found: 425.39. HRMS (ESI+) $m / z$ 448.9406 [M+Na] ${ }^{+}$, calcd. for $\mathrm{C}_{16} \mathrm{H}_{9} \mathrm{~N}_{4} \mathrm{O}_{2} \mathrm{SCl}_{3}: 448.9404[\mathrm{M}+\mathrm{Na}]^{+}$.

4-Chloro-N-(2-trichloromethylquinazolin-4-yl)benzenesulfonamide $\quad(\mathbf{8 e})$. White $\quad$ solid (50\%). M.p. $>260{ }^{\circ} \mathrm{C} .{ }^{1} \mathrm{H}-\mathrm{NMR}\left(\mathrm{DMSO}-d_{6}\right): \delta 8.25(\mathrm{~d}, J=7.6 \mathrm{~Hz}, 1 \mathrm{H}), 7.99(\mathrm{~d}, J=8.2 \mathrm{~Hz}, 2 \mathrm{H}), 7.71-7.63$ 
$(\mathrm{m}, 2 \mathrm{H}) ; 7.48-7.39(\mathrm{~m}, 3 \mathrm{H}) .{ }^{13} \mathrm{C}-\mathrm{NMR}$ (DMSO-d $\left.d_{6}\right): \delta 163.2,160.2,148.6,144.0,134.5,132.5,129.6$; 127.5, 127.0, 126.4, 125.3, 119.1, 98.8. LC-MS (ESI) Tr $2.54 \mathrm{~min}, \mathrm{~m} / z$ [M+H] ${ }^{+}$Calcd: 435.92, Found: 435.85. HRMS (ESI+) $m / z 437.9211[\mathrm{M}+\mathrm{H}]^{+}$, calcd. for $\mathrm{C}_{15} \mathrm{H}_{9} \mathrm{~N}_{3} \mathrm{O}_{2} \mathrm{SCl}_{4}: 436.9141$.

4-Bromo-N-(2-trichloromethylquinazolin-4-yl)benzenesulfonamide (8f). White solid (60\%). M.p. $>260{ }^{\circ} \mathrm{C} .{ }^{1} \mathrm{H}-\mathrm{NMR}\left(\mathrm{DMSO}-d_{6}\right): \delta 8.25(\mathrm{~d}, J=7.8 \mathrm{~Hz}, 1 \mathrm{H}), 7.92(\mathrm{~d}, J=8.2 \mathrm{~Hz}, 2 \mathrm{H}), 7.72-7.45$ $(\mathrm{m}, 5 \mathrm{H}) .{ }^{13} \mathrm{C}-\mathrm{NMR}$ (DMSO- $d_{6}$ ): $\delta 163.4,160.3,148.6,144.5,132.5,130.5,129.9 ; 127.1,126.4,125.4$, 123.3, 119.3, 98.9. LC-MS (ESI) Tr $2.60 \mathrm{~min}, \mathrm{~m} / z[\mathrm{M}+\mathrm{H}]^{+}$Calcd: 479.87, Found: 480.52. HRMS $(\mathrm{ESI}+) \mathrm{m} / z 503.8534[\mathrm{M}+\mathrm{Na}]^{+}$, calcd. for $\mathrm{C}_{15} \mathrm{H}_{9} \mathrm{BrN}_{3} \mathrm{O}_{2} \mathrm{SCl}_{3}: 503.8532[\mathrm{M}+\mathrm{Na}]^{+}$.

4-Fluoro-N-(2-trichloromethylquinazolin-4-yl)benzenesulfonamide $\quad(\mathbf{8 g})$. White solid (66\%). M.p. $>260{ }^{\circ} \mathrm{C} .{ }^{1} \mathrm{H}-\mathrm{NMR}\left(\mathrm{DMSO}-d_{6}\right): \delta 8.25(\mathrm{~d}, J=8.0 \mathrm{~Hz}, 1 \mathrm{H}), 8.10-8.03(\mathrm{~m}, 2 \mathrm{H}), 7.75-7.60(\mathrm{~m}$, 2H), $7.48(\mathrm{t}, J=7.1 \mathrm{~Hz}, 1 \mathrm{H}), 7.17(\mathrm{t}, J=8.9 \mathrm{~Hz}, 2 \mathrm{H}) .{ }^{13} \mathrm{C}-\mathrm{NMR}$ (DMSO- $\left.d_{6}\right): \delta 165.4,163.4,160.3$, 148.6, $141.5(\mathrm{~d}, J=2.9 \mathrm{~Hz}), 132.4,130.5(\mathrm{~d}, J=8.8 \mathrm{~Hz}), 127.1,126.4,125.4,119.3,114.3(\mathrm{~d}$, $J=21.8 \mathrm{~Hz}$ ), 98.9. LC-MS (ESI) Tr $1.81 \mathrm{~min}, \mathrm{~m} / z[\mathrm{M}+\mathrm{H}]^{+}$Calcd: 419.95, Found: 419.79. HRMS (ESI+) $m / z 419.9534[\mathrm{M}+\mathrm{H}]^{+}$, calcd. for $\mathrm{C}_{15} \mathrm{H}_{9} \mathrm{~N}_{3} \mathrm{O}_{2} \mathrm{SCl}_{3} \mathrm{~F}: 418.9465$.

3-Fluoro-N-(2-trichloromethylquinazolin-4-yl)benzenesulfonamide (8h). White solid (53\%). M.p. $194{ }^{\circ} \mathrm{C}$. ${ }^{1} \mathrm{H}-\mathrm{NMR}\left(\mathrm{DMSO}-d_{6}\right): \delta 8.30(\mathrm{~d}, J=8.0 \mathrm{~Hz}, 1 \mathrm{H}), 7.88(\mathrm{~d}, J=9.0 \mathrm{~Hz}, 1 \mathrm{H}), 7.77-7.65(\mathrm{~m}, 3 \mathrm{H}), 7.53(\mathrm{t}$, $J=7.8 \mathrm{~Hz}, 1 \mathrm{H}), 7.44-7.38(\mathrm{~m}, 1 \mathrm{H}), 7.25(\mathrm{t}, J=8.2 \mathrm{~Hz}, 1 \mathrm{H}) .{ }^{13} \mathrm{C}-\mathrm{NMR}$ (DMSO-d $\left.d_{6}\right): \delta 163.6,162.8$, 160.1, 158.8, 148.6, 146.8 (d, $J=6.5 \mathrm{~Hz}), 132.9,129.9$ (d, $J=7.6 \mathrm{~Hz}), 127.1$ (d, $J=21.3 \mathrm{~Hz}), 125.3$, $123.4(\mathrm{~d}, J=2.9 \mathrm{~Hz}), 118.6,117.3(\mathrm{~d}, J=20.9 \mathrm{~Hz}), 115.4(\mathrm{~d}, J=23.4 \mathrm{~Hz}), 98.5$. LC-MS (ESI) $\mathrm{Tr}$ $1.69 \mathrm{~min}, \mathrm{~m} / z[\mathrm{M}+\mathrm{H}]^{+}$Calcd: 419.95, Found: 419.81. HRMS (ESI+) $m / z 441.9359[\mathrm{M}+\mathrm{Na}]^{+}$, calcd. for $\mathrm{C}_{15} \mathrm{H}_{9} \mathrm{~N}_{3} \mathrm{O}_{2} \mathrm{SCl}_{3} \mathrm{~F}: 441.9357[\mathrm{M}+\mathrm{Na}]^{+}$.

2-Fluoro-N-(2-trichloromethylquinazolin-4-yl)benzenesulfonamide (8i). White solid (60\%). M.p. $188^{\circ} \mathrm{C}$. ${ }^{1} \mathrm{H}-\mathrm{NMR}\left(\mathrm{DMSO}-d_{6}\right): \delta 8.28(\mathrm{~d}, J=8.0 \mathrm{~Hz}, 1 \mathrm{H}), 8.06(\mathrm{dt}, J=1.5 \mathrm{~Hz}, J=7.6 \mathrm{~Hz}, 1 \mathrm{H}), 7.78-7.61(\mathrm{~m}$, 2H), $7.50(\mathrm{dt}, J=1.6 \mathrm{~Hz}, J=7.6 \mathrm{~Hz}, 1 \mathrm{H}), 7.22(\mathrm{t}, J=7.5 \mathrm{~Hz}, 1 \mathrm{H}), 7.07$ (t, $J=8.9 \mathrm{~Hz}, 1 \mathrm{H}) .{ }^{13} \mathrm{C}-\mathrm{NMR}$ (DMSO- $\left.d_{6}\right): \delta 163.4,160.3,158.0(\mathrm{~d}, J=248.8 \mathrm{~Hz}), 148.7,133.1(\mathrm{~d}, J=15.3 \mathrm{~Hz}), 132.6,132.0(\mathrm{~d}$, $J=8.1 \mathrm{~Hz}), 131.8,127.1,126.4,125.4,123.5(\mathrm{~d}, J=3.6 \mathrm{~Hz}), 119.0 ; 115.7(\mathrm{~d}, J=21.9 \mathrm{~Hz}), 98.5$. LC-MS (ESI) Tr $1.25 \mathrm{~min}, \mathrm{~m} / z[\mathrm{M}+\mathrm{H}]^{+}$Calcd: 419.95, Found: 419.80. HRMS (ESI+) $\mathrm{m} / z 441.9360$ $[\mathrm{M}+\mathrm{Na}]^{+}$, calcd. for $\mathrm{C}_{15} \mathrm{H}_{9} \mathrm{~N}_{3} \mathrm{O}_{2} \mathrm{SCl}_{3} \mathrm{~F}: 441.9357[\mathrm{M}+\mathrm{Na}]^{+}$.

4-Nitro-N-(2-trichloromethylquinazolin-4-yl)benzenesulfonamide (8j). Yellow solid (91\%). M.p. $250{ }^{\circ} \mathrm{C}$. ${ }^{1} \mathrm{H}-\mathrm{NMR}\left(\mathrm{DMSO}-d_{6}\right): \delta 8.30-8.20(\mathrm{~m}, 5 \mathrm{H}), 7.78-7.71(\mathrm{~m}, 2 \mathrm{H}), 7.51(\mathrm{~d}, J=7.2 \mathrm{~Hz}, 1 \mathrm{H}) .{ }^{13} \mathrm{C}-\mathrm{NMR}$ (DMSO- $d_{6}$ ): $\delta 163.6,160.0,151.6,148.6,148.0,132.7,128.9 ; 127.1,126.6,125.4,123.0,119.1,98.6$. LC-MS (ESI) Tr $1.87 \mathrm{~min}, \mathrm{~m} / \mathrm{z}[\mathrm{M}-\mathrm{H}]^{-}$Calcd: 444.94, Found: 445.16. HRMS (ESI+) $\mathrm{m} / z$ 468.9306 $[\mathrm{M}+\mathrm{Na}]^{+}$, calcd. for $\mathrm{C}_{15} \mathrm{H}_{9} \mathrm{~N}_{4} \mathrm{O}_{4} \mathrm{SCl}_{3}: 468.9302[\mathrm{M}+\mathrm{Na}]^{+}$.

3-Nitro-N-(2-trichloromethylquinazolin-4-yl)benzenesulfonamide (8k). Beige solid (56\%). M.p. $204{ }^{\circ} \mathrm{C}$. ${ }^{1} \mathrm{H}-\mathrm{NMR}\left(\mathrm{DMSO}-d_{6}\right): \delta 8.76(\mathrm{~s}, 1 \mathrm{H}), 8.30-8.21(\mathrm{~m}, 3 \mathrm{H}), 7.74-7.61(\mathrm{~m}, 3 \mathrm{H}), 7.51(\mathrm{t}, J=7.6 \mathrm{~Hz}, 1 \mathrm{H})$. ${ }^{13} \mathrm{C}-\mathrm{NMR}\left(\mathrm{DMSO}-d_{6}\right): \delta 163.5,160.1,148.5,147.3,147.1,133.6,132.7 ; 129.6,127.2,126.7,125.4$, 
124.6, 123.2, 119.1, 98.5. LC-MS (ESI) Tr $1.40 \mathrm{~min}, \mathrm{~m} / z$ [M-H] $]^{-}$Calcd: 444.94, Found: 445.17. HRMS (ESI+) $m / z 468.9303[\mathrm{M}+\mathrm{Na}]^{+}$, calcd. for $\mathrm{C}_{15} \mathrm{H}_{9} \mathrm{~N}_{4} \mathrm{O}_{4} \mathrm{SCl}_{3}: 468.9302[\mathrm{M}+\mathrm{Na}]^{+}$.

2-Nitro-N-(2-trichloromethylquinazolin-4-yl)benzenesulfonamide (81). Beige solid (59\%). M.p. $>260{ }^{\circ} \mathrm{C}$. ${ }^{1} \mathrm{H}-\mathrm{NMR}$ (DMSO- $d_{6}$ ): $\delta 8.34-8.25(\mathrm{~m}, 2 \mathrm{H}), 7.75-7.53(\mathrm{~m}, 6 \mathrm{H}) .{ }^{13} \mathrm{C}-\mathrm{NMR}$ (DMSO- $\left.d_{6}\right): \delta 163.7,160.2$, 148.7, 147.9, 137.3, 132.7, 131.8; 131.0, 130.7, 127.1, 126.5, 125.5, 122.2, 119.1, 98.5. LC-MS (ESI) Tr $1.16 \mathrm{~min}, \mathrm{~m} / z$ [M-H] $]^{-}$Calcd: 444.94, Found: 445.25. HRMS (ESI+) $\mathrm{m} / z$ 468.9303 [M+Na] ${ }^{+}$, calcd. for $\mathrm{C}_{15} \mathrm{H}_{9} \mathrm{~N}_{4} \mathrm{O}_{4} \mathrm{SCl}_{3}: 468.9302[\mathrm{M}+\mathrm{Na}]^{+}$.

$\mathrm{N}$-(2-trichloromethylquinazolin-4-yl)-4-(trifluoromethyl)benzenesulfonamide (8m). White solid (88\%). M.p. $166{ }^{\circ}$ C. ${ }^{1} \mathrm{H}-\mathrm{NMR}$ (DMSO- $\left.d_{6}\right): \delta 8.28(\mathrm{~d}, J=7.6 \mathrm{~Hz}, 1 \mathrm{H}), 8.13(\mathrm{~d}, J=7.2 \mathrm{~Hz}, 2 \mathrm{H}), 7.74-7.50(\mathrm{~m}$, 5H). ${ }^{13} \mathrm{C}-\mathrm{NMR}$ (DMSO- $d_{6}$ ): $\delta 163.5,160.2,149.4,148.6,132.6,129.8$ (q, $J=31.8 \mathrm{~Hz}$ ), 128.3, 127.1, 126.5, 125.4, 124.8 (q, $J=3.7 \mathrm{~Hz}$ ), 124.0 (q, $J=271.0 \mathrm{~Hz}$ ), 119.5, 98.6. LC-MS (ESI) Tr $2.80 \mathrm{~min}$, $m / z[\mathrm{M}+\mathrm{H}]^{+}$Calcd: 469.94, Found: 469.82. HRMS (ESI + ) $m / z 491.9328[\mathrm{M}+\mathrm{Na}]^{+}$, calcd. for $\mathrm{C}_{16} \mathrm{H}_{9} \mathrm{~F}_{3} \mathrm{~N}_{3} \mathrm{O}_{2} \mathrm{SCl}_{3}: 491.9325[\mathrm{M}+\mathrm{Na}]^{+}$.

$N$-(2-trichloromethylquinazolin-4-yl)-3-(trifluoromethyl)benzenesulfonamide (8n). White solid (100\%). M.p. $255{ }^{\circ} \mathrm{C} .{ }^{1} \mathrm{H}-\mathrm{NMR}$ (DMSO- $\left.d_{6}\right): \delta 8.44(\mathrm{~d}, J=8.0 \mathrm{~Hz}, 1 \mathrm{H}), 8.33-8.29(\mathrm{~m}, 2 \mathrm{H}), 7.91-7.82(\mathrm{~m}, 3 \mathrm{H})$; $7.76(\mathrm{~d}, J=8.0 \mathrm{~Hz}, 1 \mathrm{H}), 7.70-7.62(\mathrm{~m}, 1 \mathrm{H}) .{ }^{13} \mathrm{C}-\mathrm{NMR}$ (DMSO- $\left.d_{6}\right): \delta 160.6,159.3,148.7,143.9$, 134.1, 131.5, 130.0, 129.1 (q, $J=32.2 \mathrm{~Hz}), 128.4$ (q, $J=3.5 \mathrm{~Hz}), 128.0,127.8,124.7,124.4(\mathrm{q}$, $J=4.1 \mathrm{~Hz}), 123.7$ (q, $J=271 \mathrm{~Hz}), 116.6,97.5$. LC-MS (ESI) $\operatorname{Tr} 2.53 \mathrm{~min}, m / z[\mathrm{M}+\mathrm{H}]^{+}$Calcd: 469.94, Found: 469.76. HRMS (ESI+) $m / z 469.9502[\mathrm{M}+\mathrm{H}]^{+}$, calcd. for $\mathrm{C}_{16} \mathrm{H}_{9} \mathrm{~F}_{3} \mathrm{~N}_{3} \mathrm{O}_{2} \mathrm{SCl}_{3}: 468.9433$.

$N$-(2-trichloromethylquinazolin-4-yl)-2-(trifluoromethyl)benzenesulfonamide (8o). White solid (50\%). M.p. $205{ }^{\circ} \mathrm{C} .{ }^{1} \mathrm{H}-\mathrm{NMR}\left(\mathrm{DMSO}-d_{6}\right): \delta 8.71(\mathrm{~d}, J=8.2 \mathrm{~Hz}, 1 \mathrm{H}), 8.51(\mathrm{~d}, J=7.2 \mathrm{~Hz}, 1 \mathrm{H}), 8.04-7.82(\mathrm{~m}$, $6 \mathrm{H}) .{ }^{13} \mathrm{C}-\mathrm{NMR}\left(\mathrm{DMSO}-d_{6}\right): \delta 158.2,158.1,148.5,138.8,135.4,133.4,133.3,132.8,129.3,128.2$, 128.0 (q, $J=6.3 \mathrm{~Hz}$ ), 125.7 (q, $J=32.8 \mathrm{~Hz}$ ), 124.1, 122.9 (q, $J=272.4 \mathrm{~Hz}$ ), 114.2, 96.3. LC-MS (ESI) Tr $2.17 \mathrm{~min}, m / z$ [M+H] ${ }^{+}$Calcd: 469.94, Found: 469.76. HRMS (ESI+) $m / z$ 491.9327 [M+Na] calcd. for $\mathrm{C}_{16} \mathrm{H}_{9} \mathrm{~F}_{3} \mathrm{~N}_{3} \mathrm{O}_{2} \mathrm{SCl}_{3}: 491.9325[\mathrm{M}+\mathrm{Na}]^{+}$.

$N$-(2-trichloromethylquinazolin-4-yl)biphenyl-4-sulfonamide (8p). White solid (23\%). M.p. $210{ }^{\circ} \mathrm{C}$. ${ }^{1} \mathrm{H}-\mathrm{NMR}\left(\mathrm{DMSO}-d_{6}\right): \delta 8.29(\mathrm{~d}, J=7.8 \mathrm{~Hz}, 1 \mathrm{H}), 8.11(\mathrm{~d}, J=8.2 \mathrm{~Hz}, 2 \mathrm{H}), 7.73-7.65(\mathrm{~m}, 6 \mathrm{H})$, 7.62-7.36 (m, 4H). ${ }^{13} \mathrm{C}-\mathrm{NMR}$ (DMSO- $\left.d_{6}\right): \delta 163.0,160.3,148.6,143.8,141.8,139.6,132.5,129.0$, $128.5,127.8,127.1,126.9,126.5,125.9,125.4,119.1,98.9$. LC-MS (ESI) Tr $3.06 \mathrm{~min}, \mathrm{~m} / z$ [M-H] ${ }^{-}$ Calcd: 475.99, Found: 476.30. HRMS (ESI+) $m / z 499.9762[\mathrm{M}+\mathrm{Na}]^{+}$, calcd. for $\mathrm{C}_{21} \mathrm{H}_{14} \mathrm{~N}_{3} \mathrm{O}_{2} \mathrm{SCl}_{3}$ : $499.9765[\mathrm{M}+\mathrm{Na}]^{+}$.

N-(2-trichloromethylquinazolin-4-yl)naphtalene-2-sulfonamide (8q). Beige solid (43\%). M.p. $206{ }^{\circ} \mathrm{C}$. ${ }^{1} \mathrm{H}-\mathrm{NMR}$ (DMSO- $\left.d_{6}\right): \delta 8.65(\mathrm{~s}, 1 \mathrm{H}), 8.28(\mathrm{~d}, J=8.0 \mathrm{~Hz}, 1 \mathrm{H}), 8.00(\mathrm{~d}, J=8.4 \mathrm{~Hz}, 2 \mathrm{H}), 7.85(\mathrm{~d}$, $J=8.5 \mathrm{~Hz}, 2 \mathrm{H}), 7.60-7.48(\mathrm{~m}, 5 \mathrm{H}) .{ }^{13} \mathrm{C}-\mathrm{NMR}$ (DMSO- $\left.d_{6}\right): \delta 163.3,160.4,148.6,142.3,133.4,132.4$, 131.8, 128.8, 128.0, 127.5, 127.1, 126.4, 126.3, 125.4, 124.6, 119.3, 98.8. LC-MS (ESI) Tr 2.39 min, $m / z \quad[\mathrm{M}+\mathrm{H}]^{+}$Calcd: 451.97, Found: 451.79. HRMS (ESI+) $m / z 451.9785[\mathrm{M}+\mathrm{H}]^{+}$, calcd. for $\mathrm{C}_{19} \mathrm{H}_{12} \mathrm{~N}_{3} \mathrm{O}_{2} \mathrm{SCl}_{3}: 450.9716$. 
$N$-(2-trichloromethylquinazolin-4-yl)naphtalene-1-sulfonamide (8r). White solid (61\%). M.p. $>260{ }^{\circ} \mathrm{C}$. ${ }^{1} \mathrm{H}-\mathrm{NMR}\left(\mathrm{DMSO}-d_{6}\right): \delta 8.86(\mathrm{~d}, J=7.8 \mathrm{~Hz}, 1 \mathrm{H}), 8.43-8.33(\mathrm{~m}, 2 \mathrm{H}), 7.95-7.84(\mathrm{~m}, 2 \mathrm{H}), 7.68-7.42$ $(\mathrm{m}, 6 \mathrm{H}) .{ }^{13} \mathrm{C}-\mathrm{NMR}$ (DMSO- $\left.d_{6}\right): \delta 163.4,160.3,148.7,141.0,133.3,132.3,130.6,129.3,128.5,128.0$, 127.0, 126.4, 126.3, 126.1, 125.5, 125.3, 124.5, 119.1, 98.7. LC-MS (ESI) Tr $2.32 \mathrm{~min}, \mathrm{~m} / z$ [M+H] $]^{+}$ Calcd: 451.97, Found: 451.64. HRMS (ESI+) $m / z$ 473.9608 [M+Na] $]^{+}$, calcd. for $\mathrm{C}_{19} \mathrm{H}_{12} \mathrm{~N}_{3} \mathrm{O}_{2} \mathrm{SCl}_{3}$ : $473.9608[\mathrm{M}+\mathrm{Na}]^{+}$.

$\mathrm{N}$-(2-trichloromethylquinazolin-4-yl)thiophene-2-sulfonamide (8s). Beige solid (43\%). M.p. $191{ }^{\circ} \mathrm{C}$. ${ }^{1} \mathrm{H}-\mathrm{NMR}\left(\mathrm{DMSO}-d_{6}\right): \delta 8.23(\mathrm{~d}, J=8.0 \mathrm{~Hz}, 1 \mathrm{H}), 7.83(\mathrm{~d}, J=3.0 \mathrm{~Hz}, 1 \mathrm{H}), 7.77-7.62(\mathrm{~m}, 2 \mathrm{H})$, $7.57-7.45(\mathrm{~m}, 2 \mathrm{H}), 6.97(\mathrm{t}, J=4.2 \mathrm{~Hz}, 1 \mathrm{H}) .{ }^{13} \mathrm{C}-\mathrm{NMR}$ (DMSO- $\left.d_{6}\right): \delta 163.5,160.5,148.7,146.7$, $146.9,132.5,130.6,128.8,127.1,126.4,126.1,125.4,119.4,99.0$. LC-MS (ESI) Tr $1.07 \mathrm{~min}, \mathrm{~m} / z$ $[\mathrm{M}+\mathrm{H}]^{+}$Calcd: 407.91, Found: 407.86. HRMS (ESI+) $\mathrm{m} / z \quad 429.9014[\mathrm{M}+\mathrm{Na}]^{+}$, calcd. for $\mathrm{C}_{13} \mathrm{H}_{8} \mathrm{~N}_{3} \mathrm{O}_{2} \mathrm{~S}_{2} \mathrm{Cl}_{3}: 429.9016[\mathrm{M}+\mathrm{Na}]^{+}$.

$N$-(2-trichloromethylquinazolin-4-yl)methanesulfonamide (8t). White solid (18\%). M.p. $186{ }^{\circ} \mathrm{C}$. ${ }^{1} \mathrm{H}-\mathrm{NMR}\left(\mathrm{DMSO}-d_{6}\right): \delta 8.47(\mathrm{~d}, J=8.0 \mathrm{~Hz}, 1 \mathrm{H}), 7.97-7.92(\mathrm{~m}, 2 \mathrm{H}), 7.72(\mathrm{t}, J=6.2 \mathrm{~Hz}, 1 \mathrm{H}), 3.67$ (s, $3 \mathrm{H}) .{ }^{13} \mathrm{C}-\mathrm{NMR}$ (DMSO-d $): \delta 158.6,158.2,149.1,135.5,129.3,128.7,123.9,113.7,97.0,42.1$. LC-MS (ESI) Tr $0.82 \mathrm{~min}, \mathrm{~m} / z[\mathrm{M}+\mathrm{H}]^{+}$Calcd: 339.94, Found: 339.89. HRMS (ESI+) $\mathrm{m} / z$ 339.9475 $[\mathrm{M}+\mathrm{H}]^{+}$, calcd. for $\mathrm{C}_{10} \mathrm{H}_{8} \mathrm{~N}_{3} \mathrm{O}_{2} \mathrm{SCl}_{3}: 338.9403$.

\section{Conclusions}

A series of 20 quinazolines was synthetized by an efficient two-step strategy. Firstly, 4-amino-2trichloromethylquinazoline (7) was prepared in quantitative yield from the corresponding 4-chloro derivative under microwave irradiation. Then, the key amino intermediate 7 was reacted with various sulfonyl chlorides in the presence of $\mathrm{NaH}$, leading to the expected sulfonamides 8 . Synthesized compounds were highly soluble in biological media, less-toxic toward the human HepG2 cell line than previously identified hits but did not display significant in vitro antiplasmodial activity.

\section{Acknowledgments}

This work was supported by the CNRS and Aix-Marseille Université. The authors warmly thank Vincent Remusat for the ${ }^{1} \mathrm{H}$ - and ${ }^{13} \mathrm{C}$-NMR spectra recording and Marion Prieri for her contribution.

\section{References}

1. Fry, D.W.; Kraker, A.J.; McMichael, A.; Ambroso, L.A.; Nelson, J.M.; Leopold, W.R.; Connors, R.W.; Bridges, A.J. A specific inhibitor of the epidermal growth factor receptor tyrosine kinase. Science 1994, 265, 1093-1095.

2. Colotta, V.; Catarzi, D.; Varano, F.; Lenzi, O.; Filacchioni, G.; Costagli, C.; Galli, A.; Ghelardini, C.; Galeotti, N.; Gratteri, P.; et al. Structural Investigation of the 7-Chloro-3-hydroxy-1Hquinazoline-2,4-dione. Scaffold to Obtain AMPA and Kainate Receptor Selective Antagonists. Synthesis, Pharmacological, and Molecular Modeling Studies. J. Med. Chem. 2006, 49, 6015-6026. 
3. Malecki, N.; Carato, P.; Rigo, G.; Goossens, J.F.; Houssin, R.; Bailly, C.; Henichart, J.P. Synthesis of condensed quinolines and quinazolines as DNA ligands. Bioorg. Med. Chem. 2004, 12, 641-647.

4. Doyle, L.A.; Ross, D.D. Multidrug resistance mediated by the breast cancer resistance protein BCRP (ABCG2). Oncogene 2003, 22, 7340-7358.

5. Henderson, E.A.; Bavetsias, V.; Theti, D.S.; Wilson, S.C.; Clauss, R.; Jackman, A.L. Targeting the alpha-folate receptor with cyclopenta $[g]$ quinazoline-based inhibitors of thymidylate synthase. Bioorg. Med. Chem. 2006, 14, 5020-5042.

6. Foster, A.; Coffrey, H.A.; Morin, M.J.; Rastinejad, F. Pharmacological rescue of mutant p53 conformation and function. Science 1999, 286, 2507-2510.

7. Chien, T.-C.; Chen, C.-S.; Yu, F.-H.; Chern, J.-W. Nucleosides XI. Synthesis and antiviral evaluation of 5'-alkylthio-5'-deoxyquinazolinone nucleoside derivatives as $S$-adenosyl-Lhomocysteine analogs. Chem. Pharm. Bull. 2004, 52, 1422-1426.

8. Herget, T.; Freitag, M.; Morbitzer, M.; Kupfer, R.; Stamminger, T.; Marschall, M. Novel chemical class of pUL97 protein kinase-specific inhibitors with strong anticytomegaloviral activity. Antimicrob. Agents Chemother. 2004, 48, 4154-4162.

9. Martin, T.A.; Wheeler, A.G.; Majewski, R.F.; Corrigan, J.R. Sulfanilamidoquinazolines. J. Med. Chem. 1964, 7, 812-814.

10. Kunes, J.; Bazant, J.; Pour, M.; Waisser, K.; Slosarek, M.; Janota, J. Quinazoline derivatives with antitubercular activity. Farmaco 2000, 55, 725-729.

11. Waisser, K.; Gregor, J.; Dostal, H.; Kunes, J.; Kubicova, L.; Klimesova, V.; Kaustova, J. Influence of the replacement of the oxo function with the thioxo group on the antimycobacterial activity of 3-aryl-6,8-dichloro-2H-1,3-benzoxazine-2,4(3H)-diones and 3-arylquinazoline2,4(1H,3H)-diones. Farmaco 2001, 56, 803-807.

12. Gundla, R.; Kazemi, R.; Sanam, R.; Muttineni, R.; Sarma, J.A.R.P.; Dayam, R.; Neamati, N. Discovery of novel small-molecule inhibitors of human epidermal growth factor receptor-2: Combined ligand and target-based approach. J. Med. Chem. 2008, 51, 3367-3377.

13. Rewcastle, G.W.; Palmer, B.D.; Bridges, A.J.; Showalter, H.D.H.; Sun, L.; Nelson, J.; McMichael, A.; Kraker, A.J.; Fry, D.W.; Denny, W.A. Tyrosine kinase inhibitors. 9. Synthesis and evaluation of fused tricyclic quinazoline analogues as ATP site inhibitors of the tyrosine kinase activity of the epidermal growth factor receptor. J. Med. Chem. 1996, 39, 918-928.

14. Luth, A.; Lowe, W. Syntheses of 4-(indole-3-yl)quinazolines: A new class of epidermal growth factor receptor tyrosine kinase inhibitors. Eur. J. Med. Chem. 2008, 43, 1478-1488.

15. Mendes da Silva, J.F.; Walters, M.; Al-Damluji, S.; Ganellin, C.R. Molecular features of the prazosin molecule required for activation of Transport-P. Bioorg. Med. Chem. 2008, 16, 7254-7263.

16. Vanelle, P.; de Meo, M.P.; Maldonado, J.; Nouguier, R.; Crozet, M.P.; Laget, M.; Dumenil, G. Genotoxicity in oxazolidine derivatives: Influence of the nitro group. Eur. J. Med. Chem. 1990, 25, 241-250.

17. Crozet, M.P.; Giraud, L.; Sabuco, J.F.; Vanelle, P.; Barreau, M. S $\mathrm{S}_{\mathrm{RN}} 1$ reactions of a tetrasubstituted-1,4-benzoquinone. Tetrahedron Lett. 1991, 32, 4125-4128. 
18. Baraldi, P.G.; El-Kashef, H.; Farghaly, A.R.; Vanelle, P.; Fruttarolo, F. Synthesis of new pyrazolo[4,3-e]-1,2,4-triazolo[1,5-c]pyrimidines and related heterocycles. Tetrahedron 2004, 60, 5093-5104.

19. Boufatah, N.; Gellis, A.; Maldonado, J.; Vanelle, P. Efficient microwave-assisted synthesis of new sulfonylbenzimidazole-4,7-diones: Heterocyclic quinones with potential antitumor activity. Tetrahedron 2004, 60, 9131-9137.

20. Verhaeghe, P.; Azas, N.; Gasquet, M.; Hutter, S.; Ducros, C.; Laget, M.; Rault, S.; Rathelot, P.; Vanelle, P. Synthesis and antiplasmodial activity of new 4-aryl-2-trichloromethylquinazolines. Bioorg. Med. Chem. Lett. 2008, 18, 396-401.

21. Verhaeghe, P.; Azas, N.; Hutter, S.; Castera-Ducros, C.; Laget, M.; Dumètre, A.; Gasquet, M.; Reboul, J.-P.; Rault, S.; Rathelot, P.; et al. Synthesis and in vitro antiplasmodial evaluation of 4-anilino-2-trichloromethylquinazolines. Bioorg. Med. Chem. 2009, 17, 4313-4322.

22. Kabri, Y.; Azas, N.; Dumètre, A.; Hutter, S.; Laget, M.; Verhaeghe, P.; Gellis, A.; Vanelle, P. Original quinazoline derivatives displaying antiplasmodial properties. Eur. J. Med. Chem. 2010, 45, 616-622.

23. Castera-Ducros, C.; Azas, N.; Verhaeghe, P.; Hutter, S.; Garrigue, P.; Dumètre, A.; Mbatchi, L.; Laget, M.; Remusat, V.; Sifredi, F.; et al. Targeting the human malaria parasite Plasmodium falciparum: In vitro identification of a new antiplasmodial hit in 4-phenoxy-2trichloromethylquinazoline series. Eur. J. Med. Chem. 2011, 46, 4184-4191.

24. Verhaeghe, P.; Dumètre, A.; Castera-Ducros, C.; Hutter, S.; Laget, M.; Fersing, C.; Prieri, M.; Yzombard, J.; Sifredi, F.; Rault, S.; et al. 4-Thiophenoxy-2-trichloromethyquinazolines display in vitro selective antiplasmodial activity against the human malaria parasite Plasmodium falciparum. Bioorg. Med. Chem. Lett. 2011, 21, 6003-6006.

25. Augart, K.-D.; Kresze, G.; Schönberger, N. 1,6-Cyclisierungen von Phenylimino methyliminokumulenen: Bildung von Chinazolinderivaten. Justus Liebigs Ann. Chem. 1973, 1973, 1457-1466.

26. Ried, W.; Heine, B.; Merkel, W.; Kothe, N. Neuartige Synthese von 4-Tosylimino-3,4dihydrochinazolin-Derivaten. Synthesis 1976, 1976, 534-535.

27. Nordvall, G.; Yngve, U. Novel Quinazolines as 5-HT6 Modulators II. WO2007108743, 2007.

28. Yokoyama, K.; Ishikawa, N.; Igarashi, S.; Kawano, N.; Masuda, N.; Hamaguchi, W.; Yamasaki, S.; Koganemaru, Y.; Hattori, K.; Miyazaki, T.; et al. Potent and orally bioavailable CCR4 antagonists: Synthesis and structure-activity relationship study of 2-aminoquinazolines. Bioorgan. Med. Chem. 2009, 17, 64-73.

29. Verhaeghe, P.; Rathelot, P.; Gellis, A.; Rault, S.; Vanelle, P. Highly efficient microwave assisted $\alpha$-trichlorination reaction of $\alpha$-methylated nitrogen containing heterocycles. Tetrahedron 2006, 62, 8173-8176.

Sample Availability: Samples of the compounds are available from the authors.

(C) 2012 by the authors; licensee MDPI, Basel, Switzerland. This article is an open access article distributed under the terms and conditions of the Creative Commons Attribution license (http://creativecommons.org/licenses/by/3.0/). 